\section{Brass Instruments Design Using Physics-Based Sound Simulation Models and Surrogate-Assisted Derivative-Free Optimization}

\begin{abstract}
This paper presents a method for design optimization of brass wind instruments. The shape of a trumpet's bore is optimized to improve intonation using a physics-based sound simulation model. This physics-based model consists of an acoustic model of the resonator, a mechanical model of the excitator, and a model of the coupling between the excitator and the resonator The harmonic balance technique allows the computation of sounds in a permanent regime, representative of the shape of the resonator according to control parameters of the virtual musician. An optimization problem is formulated in which the objective function to be minimized is the overall quality of the intonation of the different notes played by the instrument. The design variables are the physical dimensions of the resonator. Given the computationally expensive function evaluation and the unavailability of gradients, a surrogateassisted optimization framework is implemented using the mesh adaptive direct search algorithm (MADS). Surrogate models are used both to obtain promising candidates in the search step of MADS and to rank-order additional candidates generated by the poll step of MADS. The physics-based model is then used to determine the next design iterate. Two examples (with two and five design optimization variables) demonstrate the approach. Results show that significant improvement of intonation can be achieved at reasonable computational cost. Finally, the perspectives of this approach for computer-aided instrument design are evoked, considering optimization algorithm improvements and problem formulation modifications using for instance different design variables, multiple objectives and constraints or objective functions based on the instrument's timbre.
\end{abstract}

Robin Tournemenne

Institut de Recherche en Communications

et Cybernétique de Nantes, UMR CNRS 6597

École Centrale Nantes,

1 rue de la Noë,

Nantes 44300, France

e-mail: robin.tournemenne@irccyn.ec-nantes.fr

Jean-François Petiot

Institut de Recherche en Communications et

Cybernétique de Nantes,

UMR CNRS 6597

École Centrale Nantes

1 rue de la Noë,

Nantes 44300, France

e-mail: jean-francois.petiot@irccyn.ec-nantes.fr

Bastien Talgorn

Department of Mechanical Engineering, GERAD and McGill University,

Montréal, QC H3T1J4, Canada

e-mail: bastien.talgorn@mail.mcgill.ca

Michael Kokkolaras

Department of Mechanical Engineering, GERAD and McGill University,

Montréal, QC H3T1J4, Canada e-mail: michael.kokkolaras@mcgill.ca

Joël Gilbert

Laboratoire d'Acoustique

de l'Université du Maine,

UMR CNRS 6613

Université du Maine,

Le Mans 72085, France

e-mail: joel.gilbert@univ-lemans.fr

\section{Introduction}

The study of sound quality and playing properties of musical instruments is critical to improving the design of the latter. This is not a trivial task because sound quality is influenced by objective acoustic characteristics of the instrument and subjective criteria related to players' feelings, tastes, and preferences. Two main kinds of studies aim at addressing this issue. On the one hand, the quality can be assessed by listeners or players (subjective quality) during evaluation tests [1]. When conducting this kind of tests, it is necessary for the researchers to control precisely the design parameters of the instrument and the testing environment, to assess the reliability of the evaluations, and to define how to deal with interindividual differences. On the other hand, the quality can be quantified by physical measurements on the instruments (objective quality) [2].

For the brass instruments considered in this paper, the single most important physical measurement that characterizes behavior is the acoustic input impedance of the resonator [2]: it yields the magnitude of the acoustic response to a forced oscillation. It can be either measured or computed with an acoustic model [3], and is a function of the inner shape of the pipe (called the "bore"). For example, the typical input impedance of a trumpet presents several peaks of impedance that represent the acoustic resonances of the pipe (see Fig. 1).

When playing, the musician produces a sound whose frequency (i.e., the playing frequency) is close to the resonance frequency of an impedance peak [4]. At first approximation, the playing frequency (which influences intonation) is mainly governed by the corresponding peak of impedance. The challenge for an instrument maker is to define a bore that will produce notes with precise intonation.

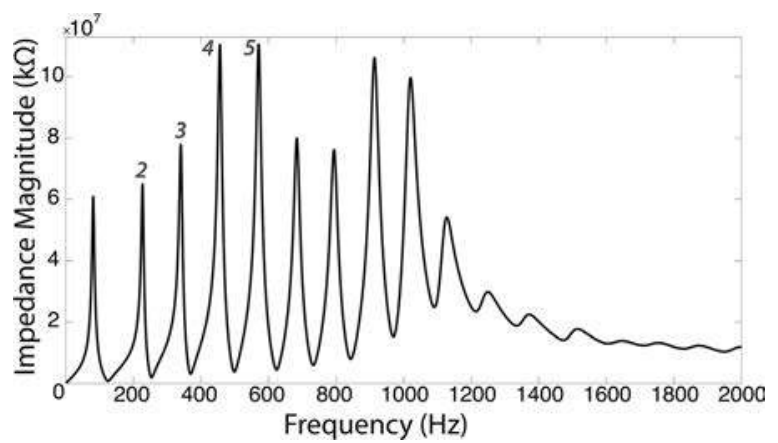

Fig. 1 Typical input impedance $Z$ of a $B b$ trumpet (magnitude), showing the resonances $2,3,4,5$ of the instrument 
With this in view, many researchers have used an optimization approach to design an instrument's inner shape with a given set of properties concerning input impedance. Kausel used genetic algorithms and the Rosenbrock minimization procedure to optimize the intonation of brass instruments [5]. Different criteria for bore optimization of the trombone can be found in Ref. [6]. Noreland used gradient-based algorithms to optimize the intonation of horns using a model for impedance calculation that combines a one-dimensional transmission line model with a two-dimensional finite element model [7]. Poirson et al. propose the integration of subjective and objective assessments of designs into the optimization process [8]. A perceptual study has been conducted on a set of trumpets to define the target frequency ratio for the resonances of impedance.

However, even if valuable, these approaches focus only on the performance of the instrument alone, neglecting a crucial element in playing an instrument: the musician. The studies of Eveno et al. showed in particular that the relations between the resonance frequencies of the impedance and the actual frequencies of the sounds played by musicians can be significantly different [9]. Although interesting information can be given by the impedance concerning the intonation of an instrument, it is still very difficult to predict the "playability" and sound qualities of brass only based on the impedance.

A second approach in the characterization of a musical instrument is the use of a physics-based model that models not only the instrument, but also its interaction with the musician. In this context, sound simulations by physics-based modeling are interesting because they can simulate the function of instruments in a realistic way, as far as the underlying physics are captured adequately [10]. For example, time domain simulations are presented in Ref. [11], where the authors investigate perceptual differences between simulated guitar sounds obtained by modifications of the mechanical parameters of the body. Sound simulations by physics-based modeling constitute a promising means to study and improve the function of musical instruments, but their use is limited when it comes to instrument design [2].

The main objective of this work is to present a new paradigm for the design and optimization of brass musical instruments. The novelty of the approach lies in the integration of sound simulations, obtained from a physics-based model that takes into account the interaction of the instrument with a virtual musician, into the optimization process. To illustrate the approach, we will demonstrate it on a particular brass instrument, the trumpet. The implemented simulation method (the harmonic balance technique [12]) allows the computation of sounds in a permanent regime (autooscillations), which is representative of the shape of the resonator according to control parameters of the virtual musician embouchure (the mechanical parameters of the excitatory system). Playing frequencies and the spectra of different notes of the tessitura of a trumpet can then be characterized using these simulations. Various virtual embouchures, which lead to convergence of the system toward auto-oscillations, are considered to produce simulations that are representative of the resonator. Due to the high computational cost of this approach and the difficulty to estimate gradients due to the stochastic nature of the objective function of the optimization problem, a surrogate-assisted optimization framework that utilizes the derivative-free mesh-adaptive direct search (MADS) algorithm is adopted.

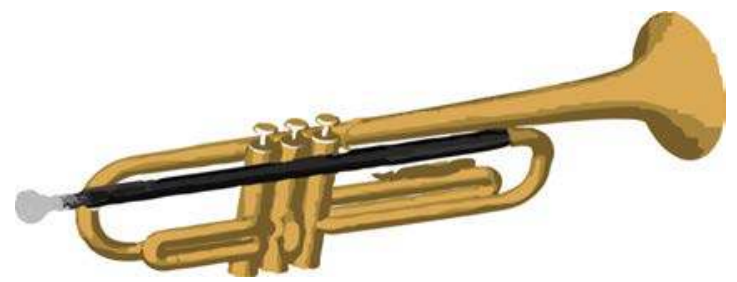

Fig. 2 Definition of the main parts of the trumpet: the mouthpiece (in light gray), the leadpipe (in black), and the flaring bell
The paper is organized as follows: We first present extended details about the trumpet's function, the physics-based brass model, and the simulation technique to clarify the sound simulation method. We then formulate the optimization problem and describe the principles of the MADS algorithm and the framework for surrogate-assisted optimization. Finally, we conduct two case studies concerning the shape optimization of trumpets with two and five design variables, respectively, and draw conclusions.

\section{Trumpet Modeling}

Brass instruments are wind instruments that produce sounds by the coupling of an excitator (the lips of the musician) to a resonator (the body of the instrument). The main parts of the resonator are the mouthpiece (a short removable piece of metal on which the musicians place their lips), the leadpipe (a roughly conical part, essential to the intonation of the instrument), and the flaring bell (see Fig. 2). Three valves are necessary to adjust the length of the resonator and to obtain chromatic scales.

The characteristics of a played note and its timbre depend mainly on the inner shape of the resonator (the bore) and, of course, on the musician's technical ability and skill. From a pressure $P_{m}$ in the mouth of the musician (typical values measured on trumpet players are from $1 \mathrm{kPa}$ to about $12 \mathrm{kPa}$ ), the lips act as a vibrating valve that modulates the air flow into the instrument [2]. The column of air in the instrument vibrates, according to the resonance frequencies of the resonator. A regime of oscillations is created as a result of the complex coupling between the resonator and the lips. It is important to mention that this coupling is the result of the reaction of the resonator on the lips: vibrations of the lips are facilitated at frequencies which correspond to the resonance frequencies of the bore. With the same bore, several notes can be obtained, corresponding to different regimes of oscillations governed by the resonance frequencies. Given that occidental music is globally written with 12 different notes per octave which is called the chromatic scale, one bore representing roughly 8 exploitable notes is not enough. In order to cope with this issue three cylindrical valves placed in the middle of the instrument (see Fig. 2) lengthened the bore shifting the resonances toward lower frequencies. Finally, using the right combination of the three valves and the right embouchure, the musician can produce more than 12 different notes over 3 octaves $(3 * 12=36$ notes).

From an acoustic point of view, the resonator alone can be characterized by its input impedance $Z$, showing the resonance frequencies of the bore (see Fig. 1). It represents the response of the instrument in forced oscillations for a given frequency range and shows several peaks (corresponding to resonances) that are used to play the different notes. $Z$ can be either measured on a real instrument [13] or modeled using an acoustic model of the wave propagation in the resonator. From the definition of the geometry of the resonator, the input impedance can be computed using the transmission line modeling [3]. This model considers the inner shape of the instrument as a concatenation of simple geometries as cylinders,

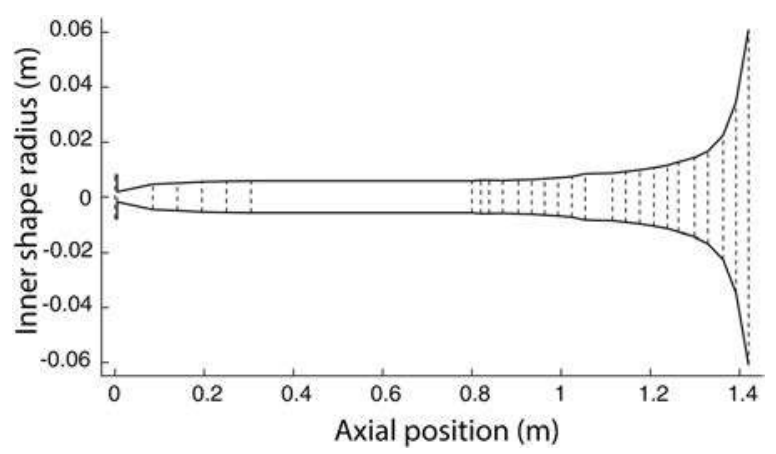

Fig. 3 Bore geometry of a trumpet, described as a series of conical and cylindrical segments 
cones, Bessel horns, exponential horns, and discontinuities (see Fig. 3 for an example of the bore geometry of a trumpet), for which the expressions of the input impedance are explicit with a transfer matrix modeling. The input impedance of the entire instrument is finally computed by taking the product of the matrices of all the elements (transfer matrix of a horn). The model considered in this paper takes into account plane-wave propagation and visco-thermal losses, but more advanced models with bents, wall energy losses, and multimodal approaches for flaring bells could be utilized.

2.1 Physics-Based Model. The physics-based model of brass instruments available in the literature is given by

$$
\begin{gathered}
p(j \omega)=Z(j \omega) v(j \omega) \\
\frac{d^{2} H(t)}{d t^{2}}+\frac{2 \pi f_{l}}{Q_{l}} \frac{d H(t)}{d t}+\left(2 \pi f_{l}\right)^{2}\left(H(t)-H_{0}\right)=\frac{P_{m}-p(t)}{\mu_{l}} \\
\text { and } v(t)=b H(t) \sqrt{\frac{2\left(P_{m}-p(t)\right)}{\rho}}
\end{gathered}
$$

While this work considers trumpets, the same model can be employed for any reed instrument. In general, the input impedance paradigm is commonly used for wind instruments. Moreover, this approach can be extended to any auto-oscillating musical instrument, such as bowed strings, flutes/organs, human voices, and, of course, other brass and reed instruments. These three equations represent the behavior of the different parts of the system [2]. They couple three time-varying variables: the opening height $H(t)$ of the two lips, the volume flow $v(t)$ and the pressure $p(t)$, in the mouthpiece. Equation (1), with the frequency domain notation, describes the acoustic behavior of the resonator. It represents the impedance $Z$ of the instrument, defined as the ratio of acoustic pressure in the mouthpiece $p(j \omega)$ to the acoustic volume flow $v(j \omega)$ entering the instrument. Equation (2) describes the mechanical behavior of the lips of the musician considered as a mechanical oscillator with one degree-of-freedom. Said degree-of-freedom is the opening height $H(t)$ between the two lips $(H(t)=0$ is numerically imposed if $H(t)<0$ ). Equation (3) describes the coupling between the lips and the trumpet. Obtained by expressing the Bernoulli theorem, it represents a nonlinear coupling between the pressure in the mouthpiece $p(t)$, the opening height of the lips $H(t)$, and the volume flow $v(t)$.

While more elaborate models may be developed by refining the description of the system, the model presented above is sufficient to characterize the underlying physics and the sound perception of brass instruments. References [10] and [14] examine the perceptual differences in the simulated sound of different trumpets, producing sounds using the model of Eqs. (1)-(3). When the dimensions of two instruments differ significantly (order of millimeters), human participants are able to distinguish among simulated sounds. When the dimensions are very similar (order of some tenths of $\mathrm{mm}$ ), different simulated sounds cannot be distinguished by the participants.

Several parameters are introduced in this model: (1) the parameters concerning the musician's embouchure are $P_{m}$ (the pressure in the mouth), $f_{l}$ (the resonance frequency of the lips), $\mu_{l}$ (the area density of the lips), $b$ (the width of the lips), $H_{0}$ (the rest value of the opening height of the lips), and $Q_{l}$ (the quality factor of the resonance of the lips); (2) the input impedance $Z$ of the trumpet; and (3) the air density $\rho$. Numerical solutions $p(t)$ of this system of equations can be computed to simulate the sound created by a given trumpet (defined by its input impedance $Z$ ) and for a given "virtual musician embouchure" (defined by its control parameters).

2.2 Simulations Using the Harmonic Balance Technique. The harmonic balance technique is a particular method used to obtain numerical solutions of the physics-based model described above. This technique simulates sounds in a permanent regime (steady state) in the frequency domain. The principle is to compute (if it exists) a converging periodic solution of the pressure $p(t)$ of the system, taking into account a given finite number $N$ of harmonics in a truncated Fourier series (Eq. (4))

$$
p(t)=\sum_{n=1}^{N} A_{n} \cos \left(2 \pi n F t+\varphi_{n}\right)
$$

Assuming that the solution $p(t)$ of the system of equations is harmonic, the unknown values of the simulations are the amplitudes of the harmonics $A_{n}$, the phases $\varphi_{n}$, and the playing frequency $F$. A numerical solution $p(t)$ of the auto-oscillating system satisfying Eqs. (1)-(3) can be defined if the system converges toward a stable solution (more details can be found in Ref. [12]).

2.3 Control Parameters of the Simulations. To perform a sound simulation, it is necessary to define the relevant values (i.e., the values that lead to a convergence toward a steady-state sound for a given note) for the parameters of the musician embouchure (in other words, it is necessary to "teach" the computer how to play the trumpet). For a given note, the experience shows that many embouchures may lead to a steady-state note. The choice of the ranges of the parameters is based both on numerical tests of the simulations and on measurements on real trumpet players. In this study, the values of $b, Q_{l}, H_{0}$ are considered as fixed [15]. The three variables $P_{m}, \mu_{l}$, and $f_{l}$ are considered as control parameters of the simulations, and constitute the virtual embouchure. As for a real trumpet player, the pressure $P_{m}$ in the mouth influences mainly the dynamics of a simulated sound. Since experimental measurements on real musicians provided maximum values around $12 \mathrm{kPa}$ for the pressure in the mouth [16], the range of values selected in this study runs from $6 \mathrm{kPa}$ to $9 \mathrm{kPa}$, which corresponds roughly to mezzoforte $(m f)$ dynamics. The values of $\mu_{l}$ range from 1 to $6 \mathrm{~kg} / \mathrm{m}^{2}$ [15]. Finally, the frequency of the lips $f_{l}$ is the parameter that allows the selection of the played regime (note): the higher the value of $f_{l}$, the higher the simulated regime. Exploration tests led to a range of $f_{l}$ that spans from $130 \mathrm{~Hz}$ to $480 \mathrm{~Hz}$ to simulate the second, third, fourth, and fifth regimes of the $\mathrm{Bb}$ trumpet with no valve pressed, the regimes considered in this study. These regimes correspond to the musical notes Bb3, F4, Bb4, D5-concert-pitch, see Fig. 4.

The values of the control parameters considered in this study are given in Table 1 . These values were selected because they generally lead to a convergence of the auto-oscillations toward a periodic solution.

Given that the impedance model is limited in frequency (see Fig. 1), it is not relevant to consider many harmonics for the sound simulation. Above $3000 \mathrm{~Hz}$, the magnitude of the impedance is flat and no difference between trumpets is noticeable. Therefore, the number $N$ of harmonics considered for the simulations has been bounded to $3000 \mathrm{~Hz}$. For the two highest notes, Bb4 $(466.16 \mathrm{~Hz})$ and D5 $(587.33 \mathrm{~Hz})$, this allows roughly the computation of five or six harmonics. All the sounds have thus been simulated with $N=6$ harmonics. In conclusion, for a given trumpet (characterized by its input impedance $Z$ ) and for a virtual musician embouchure (characterized by the parameters $P_{m}, \mu_{l}, f_{l}, b, H_{0}$, and $Q_{l}$ ), the simulations generate one note, corresponding to one of the regimes, 2, 3, 4, and 5, of the trumpet. Each note is characterized by its playing frequency $F$ and by the amplitudes and phases of its 6 first harmonics.

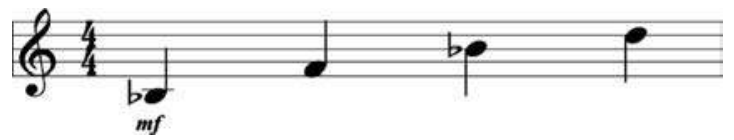

Fig. 4 Musical notation of the notes $\mathrm{Bb3}, \mathrm{F} 4, \mathrm{Bb} 4$, and $\mathrm{D} 5$ of the $\mathrm{Bb}$ trumpet that are simulated in this study 


\section{Optimization Problem Formulation}

The design optimization problem of an instrument can be formulated as

$$
\min _{\mathbf{x} \in \Omega} J(\mathbf{x})
$$

where $J: \mathbb{R}^{n} \rightarrow \mathbb{R}$ is the objective function, and $\mathbf{x}$ represents the design parameters of the instrument. The design space $\Omega$ is a subset of $\mathbb{R}^{n}$ delimited by box (bound) constraints. Specifically, the optimization variables are the geometric parameters that define the inner shape of the bore (see Fig. 3). To facilitate the input impedance calculations, the bore is approximated by a series of conical and cylindrical waveguide segments. Consequently, $\mathbf{x}$ is a vector of geometric quantities such as the lengths and radii of cylinders or cones. $\Omega$ corresponds to constraints applied on $\mathbf{x}$ to obtain viable trumpet shapes.

In this work, we estimate the quality of an instrument by its intonation. Other possible descriptors (based on timbre or volume) can be found in the literature; however, there is no consensus in the community regarding the quality of sounds. These disagreements notwithstanding, there is a strong consensus that a trumpet playing in tune is desirable. Therefore, our objective function $J$ is a descriptor of the instrument's intonation: it is computed from the playing frequencies $F$ produced by the simulations for the different regimes of the instrument (see Fig. 5).

3.1 Computation of the Objective Function. The flowchart of the process for optimizing the shape of a trumpet bore using physics-based sound simulations is depicted in Fig. 5. The input impedance is computed based on the design vector $\mathbf{x}$ representing the resonator's geometry. The harmonic balance technique simulates $P$ different notes based on the calculated input impedance $Z(\mathbf{x})$ and some virtual embouchures chosen in a set $\varphi$ of possible embouchures (see next paragraph for their definition). While various virtual embouchures are considered, only the ones that lead to convergence of the system toward auto-oscillations are selected. This selection process is a crucial step in the method and is described in more detail in the next paragraph.

For each note $i$, the average (across different embouchures) playing frequency $\overline{F_{i}}(\mathbf{x}, \varphi)$ is computed. The intonation of the note is assessed by the deviation of the expected playing frequency from the actual playing frequency as simulated using the physics-based model. To compute this deviation, the cent logarithmic unit is used because it is standard to compare musical pitches (there are no scaling issues), given that human perception of pitches is based on frequency ratios ( 1 cent corresponds to a frequency ratio equal to a hundredth of a semitone). To compute this deviation, a reference (tuning) note and a reference scale (considered as the correct intonation) are necessary. Trumpet players tune generally their instrument on the fourth regime of the trumpet with no valve pressed (Bb4, concert pitch), so it is used as the reference note.

The equal-tempered scale (which means that the octave is divided in 12 equal semi-tones) is chosen as the reference scale, given its worldwide use in occidental music. While it is possible to consider customized musical temperament for a particular

Table 1 Values of the control parameters for the simulations considered in the study (virtual musician embouchure)

\begin{tabular}{lcc}
\hline \hline Definition & Notation & Value \\
\hline Resonance frequency of the lips & $f_{l}(\mathrm{~Hz})$ & $130-480$ \\
Mass per area of the lips & $\mu_{l}\left(\mathrm{~kg} / \mathrm{m}^{2}\right)$ & $1-6$ \\
Pressure in the mouth & $P_{m}(\mathrm{kPa})$ & $6-9$ \\
Width of the lips & $b(\mathrm{~mm})$ & 10 \\
Rest value of the opening height & $H_{0}(\mathrm{~mm})$ & 0.1 \\
Quality factor of the resonance & $Q_{l}$ & 3 \\
\hline \hline
\end{tabular}

trumpet player, such consideration is beyond the scope of this paper. For every note $i$, the equal-tempered deviation (ETD) between the average frequency of the $i$ th note, $\overline{F_{i}}(\mathbf{x}, \varphi)$, and the reference frequency $\overline{F_{\text {ref }}}(\mathbf{x}, \varphi)$ is given by

$$
\left.\operatorname{ETD}(\mathbf{x}, i, \varphi)=\alpha_{\text {ref } \rightarrow i}-1200 \log _{2} \frac{\overline{F_{i}(\mathbf{x}, \varphi)}}{\overline{F_{\text {ref }}(\mathbf{x}, \varphi)}}\right)
$$

where $\alpha_{\mathrm{ref} \rightarrow i}$ is the difference between the reference note ref and the targeted note $i$ given by the equal-tempered scale ( -500 cents for example between $\mathrm{Bb} 4$ and $\mathrm{F} 4$ between which the interval is a descending fourth). The objective function $J(\mathbf{x}, \varphi)$ for the whole instrument is the average of the absolute deviation across the $(P-1)$ notes (note that the deviation between the reference note and the fourth note is always equal to zero)

$$
J(\mathbf{x}, \varphi)=\frac{1}{P-1} \sum_{i \in \text { Notes }}|\operatorname{ETD}(\mathbf{x}, i, \varphi)|
$$

The main challenge in the proposed approach lies in the convergence of the simulations toward auto-oscillations. This refers to the instability of nonlinear systems for which no analytical solutions are available. For a given instrument geometry, appropriate virtual embouchures must be selected to ensure that the simulations converge toward a sound in a permanent regime. Furthermore, similar to an inexperienced player that would blow a trumpet with a terrible sound, the virtual embouchure must be carefully selected in order to produce realistic sounds. A preprocessing of the simulations is thus necessary to obtain, for different geometries of instruments, a set of appropriate embouchures that converge toward realistic sounds. To that end, a criterion has been defined to represent the amplitude of the simulated sound relatively to the pressure in the mouth. If the amplitudes of the harmonics are large enough relative to the mouth pressure $P_{m}$ produced by the virtual musician, the embouchure is considered appropriate. For a given note, a sound is considered realistic if

$$
\frac{\sqrt{\sum_{i=1}^{6} A_{i}^{2}}}{P_{m}} \geq \text { Threshold }
$$

An exploration of the design space according to the embouchure parameters is carried out to determine the threshold for each note and to build a map of appropriate embouchure-geometry couples. If $\mathbf{x}$ is in $\mathbb{R}^{2}$, the space to explore has five dimensions: two geometric variables and three embouchure variables $\left(P_{m}, \mu_{l}\right.$, $f_{l}$ ). To explore this space, a five-dimensional Latin hypercube is built and every sample is simulated. This constitutes the preprocessing of the parameters of the simulations. The thresholds are adjusted to select $10 \%$ of the simulated sounds for a given note. A map of appropriate embouchure-geometry couples is drawn from the previously selected sounds (using a Gaussian mixture model that fits the distribution).

It is important to mention that the average playing frequency, $\overline{F_{i}}(\mathbf{x}, \varphi)$, is an average value across a finite set of embouchures. Furthermore, this set of embouchures is not deterministic, given

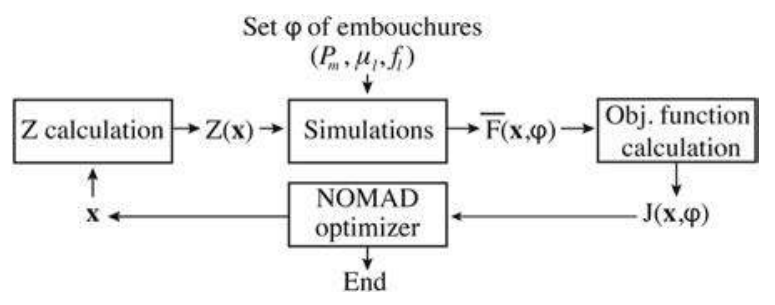

Fig. 5 Flowchart of the optimization process 
that the preprocessing determination of appropriate embouchures is stochastic (random sampling using the Gaussian mixture model). The consequence is that the objective function $J(\mathbf{x})$ is not deterministic (two consecutive calculations may give different results). In practice, a set of 100 embouchures is simulated to provide an estimation of $\overline{F_{i}}(\mathbf{x}, \varphi)$ for one note.

\section{Surrogate-Assisted Derivative-Free Optimization Methodology}

Since the objective function is the result of a stochastic simulation, derivatives are not available and cannot be approximated reliably. Therefore, we use a rigorous derivative-free algorithm with convergence properties, MADS [17-19], available via the NOMAD software. This algorithm is based on the search-and-poll paradigm introduced in Ref. [20]. The search step can implement any user-defined method to obtain promising candidates. The poll step determines candidates around the incumbent solution; it ensures the convergence of the algorithm toward a local optimum. In this work, we use a variation of the surrogate-assisted optimization framework proposed in Ref. [21]. The basic idea is that we use surrogate models of the "true" physics-based sound simulation model to (i) formulate and solve a surrogate problem in the search step to obtain a promising candidate and (ii) rank-order the candidates generated by the poll step. We then use the "true" physicsbased sound simulation model to evaluate opportunistically all these candidates in order to determine the next iterate.

4.1 Mesh Adaptive Direct Search. At each iteration $k$ of the MADS algorithm, the trial points must lie on a mesh $M_{k}$ defined as

$$
M_{k}=\left\{\mathbf{x}+\Delta_{k}^{m} D \mathbf{z}: \mathbf{z} \in \mathbb{N}^{n_{D}}, \mathbf{x} \in \mathbf{X}_{k}\right\} \subset \mathbb{R}^{n}
$$

where $\Delta_{k}^{m}$ is the mesh size parameter, the columns of $D \in \mathbb{R}^{n \times n_{D}}$ form a positive spanning set of $n_{D}$ directions in $\mathbb{R}^{n}$ [19], and $\mathbf{X}_{k}=\left\{\mathbf{x}_{1}, \mathbf{x}_{2}, \ldots, \mathbf{x}_{p}\right\} \subset \mathbb{R}^{n}$ denotes the set of points already evaluated.

During each search step, an ensemble of surrogate models of the "true" physics-based sound simulation model use to evaluate the objective function $J$ is built using previous evaluations. The considered ensemble of surrogate models is described in section 4.2. The best surrogate model is selected, and a second instance of MADS is used to obtain the design that minimizes it. This instance of MADS uses two starting points: the incumbent solution of the problem, and (if available) the point returned by the previous search. This design is then projected on the mesh $M_{k}$ and evaluated using the "true" physics-based sound simulation model. If this candidate leads to an improvement of the solution, the surrogate model ensemble is updated and the search is repeated. Otherwise, the algorithm continues with the poll step.

During each poll step, the poll set is defined as $P_{k}=\left\{\mathbf{x}_{k}+\Delta_{k}^{p} \mathbf{d}, \mathbf{d} \in D_{k}\right\}$, where $D_{k}$ is a set of normalized directions such that these directions are positively spanning of $\mathbb{R}^{n}$ such that $P_{k} \subset M_{k}$; the interested reader can refer to Ref. [17] for
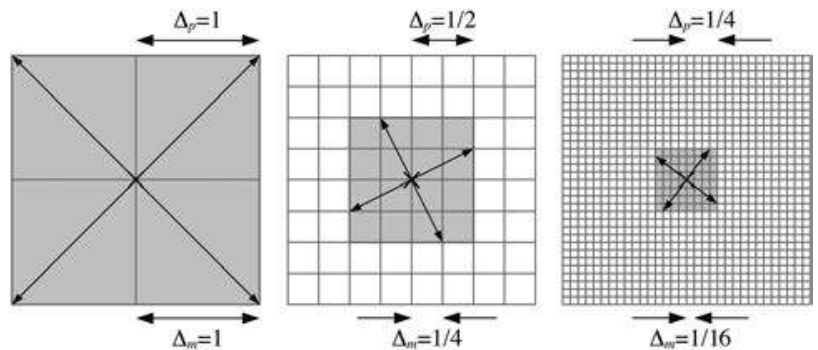

Fig. 6 MADS poll and mesh sizes for a two-dimensional problem details. The poll set is then sorted using the surrogate model, and the points are evaluated using the "true" physics-based sound simulation model in an opportunistic manner, which means that the evaluation of $P_{k}$ is interrupted if a candidate leads to an improvement of the solution. In this case, the mesh and poll size parameters are increased so that the algorithm progresses faster toward a better solution. Otherwise, these parameters are reduced, which means that the next poll will look for trial points in a closer neighborhood. A fundamental aspect of the MADS algorithm is that the mesh size decreases faster than the poll size, which means that the set of polling direction becomes dense in $\mathbb{R}^{n}$ once normalized (see Fig. 6).

4.2 Surrogate Models. To build a robust and accurate surrogate model of the "true" objective function, we rely on an ensemble of surrogate models [22-24]. At the beginning of each search step, 17 different surrogate models (see Table 2) are built. For each of these models, an error metric is computed to enable the selection of the best model at the beginning of each search or poll step. The selected model is then used in the search step to provide an interesting candidate and in the poll step to order the poll candidates. An alternative to the use of ensembles of surrogates is the selection of one type of model and the optimization of its parameters. The selection of the best surrogate type, however, can be difficult to do in advance: in part because of the variety of options available, and in part because the most suitable type may change as the optimization process unfolds. Moreover, the optimization of the parameters of the surrogate may be computationally expensive. In Ref. [25], we showed that the use of ensembles of models is a robust and efficient approach for surrogate-assisted optimization.

The rationale behind updating all surrogates at every iteration is that the best model can vary. The surrogates are built using three different modeling techniques: Polynomial response surfaces (PRSs) [24,26,27], Kernel smoothing (KS) [24,28], and radial basis functions (RBFs) $[24,26,27,29,30]$. For each of these modeling methods, previously generated data $[\mathbf{X}, J(\mathbf{X})]$ are used to build a surrogate $\hat{J}$ of the function $J$.

4.2.1 Polynomial Response Surfaces. A PRS model is a linear composition of polynomial basis functions

$$
\hat{J}(\mathbf{x})=\sum_{j=1}^{q} c_{j} h_{j}^{\mathrm{PRS}}(\mathbf{x})
$$

where the coefficients $\mathbf{c}=\left\{c_{j}\right\}_{j=1, \ldots, q} \in \mathbb{R}^{q}$ are computed by ordinary least squares (OLS) to minimize

Table 2 List of surrogate models built during the search step

\begin{tabular}{cccccc}
\hline \hline$\#$ & $\begin{array}{c}\text { Model } \\
\text { type }\end{array}$ & Degree & $\begin{array}{c}\text { Ridge } \\
\text { param. } r\end{array}$ & $\begin{array}{c}\text { Shape } \\
\text { param. } \lambda\end{array}$ & $\begin{array}{c}\text { Kernel } \\
\phi(d)\end{array}$ \\
\hline 1 & PRS & 1 & 0 & N.A. & N.A. \\
2 & & 1 & 0.001 & & \\
3 & & 2 & 0 & & \\
4 & & 2 & 0.001 & & \\
5 & & 3 & 0 & & \\
6 & & 6 & 0.001 & & \\
7 & KS & N.A. & N.A. & 0.1 & $e^{-\lambda^{2} d^{2}}$ \\
8 & & & & 0.3 & \\
9 & & & & 1.0 & \\
10 & & & & 3.0 & \\
11 & & & & 10.0 & \\
12 & RBF & 1 & 0.001 & 0.3 & $e^{-\lambda^{2} d^{2}}$ \\
13 & & & & 1.0 & \\
14 & & & & 3.0 & \\
15 & & & & N.A. & $d$ \\
16 & & & & & $d \log (d)$ \\
17 & & & & & \\
\hline \hline
\end{tabular}




$$
\sum_{i=1}^{p}\left(J\left(\mathbf{x}_{i}\right)-\hat{J}\left(\mathbf{x}_{i}\right)\right)^{2}+r \sum_{j=1}^{q} c_{j}^{2}
$$

where $r$ is a ridge parameter (typically, $r=0.001$ ), which allows to regularize the OLS system, especially when the number of training points is smaller than the number of basis functions. The degree of the PRS defines the maximum degree of the polynomials $\left\{h_{j}^{\mathrm{PRS}}\right\}_{j=1, \ldots, q}$, which are chosen to form a basis of the polynomial vector space for that given degree.

Since the objective function is not smooth, a PRS of high degree may have a tendency to over-fit the data. In other words, a higher PRS degree may lead to a higher cross-validation error. The ridge parameter can be fine-tuned to minimize crossvalidation error, but this process is time-consuming. In this study, the accuracy of the surrogate is attained by building several models rather than by fine-tuning their parameters. For this reason, surrogates 1-6 in Table 2 are PRS models of various degrees and ridge parameter values.

4.2.2 Radial Basis Functions. RBF models are linear combinations of PRS basis functions and radial basis functions

$$
\hat{J}(\mathbf{x})=\sum_{j=1}^{q^{\mathrm{PRS}}} c_{j}^{\mathrm{PRS}} h_{j}^{\mathrm{PRS}}(\mathbf{x})+\sum_{j=1}^{q^{\mathrm{RBF}}} c_{j}^{\mathrm{RBF}} h_{j}^{\mathrm{RBF}}(\mathbf{x})
$$

Unlike most RBF model formulations [24,29,30], $q^{\mathrm{RBF}}$ is chosen so that $q^{\mathrm{RBF}}+q^{\mathrm{PRS}} \leq p$, which means that there is not one radial basis function per training point, and that this model does not necessarily match the value of $J$ at the training points. However, this allows to limit the number of basis functions and the time necessary to compute the coefficients of the model. As for PRS models, these coefficients are computed by minimizing the error described in Eq. (11). In particular, when the number of training points is smaller than the number of basis functions, the regularization term is required to build the model. Consequently, for RBF models, the ridge parameter and the degree of the PRS must be specified as parameters of the model (see Table 2). In this work, the degree of the PRS in an RBF model is always one. The function $h_{j}^{\mathrm{RBF}}$ is radial basis function of the form $h_{j}^{\mathrm{RBF}}(\mathbf{x})=\phi\left(\left\|\mathbf{x}, \mathbf{x}_{j}^{s}\right\|\right)$ where the points $\left\{\mathbf{x}_{j}^{s}\right\}_{j=1, \ldots, q^{\mathrm{RBF}}}$ are a subset of $\mathbf{X}$ selected greedily to maximize the distance between the points within this subset. The function $\phi(d)$ is either a Gaussian kernel of shape parameter $\lambda$ (models $7-10$ ) or an harmonic spline of order 1 or 2 (models 11 and 12 , respectively) [29].

4.2.3 Kernel Smoothing. The Kernel Smoothing prediction in $\mathbf{x}$ is a weighted average of all observations $J(\mathbf{X})$, where the weight assigned to an observation decreases when its distance from $\mathbf{x}$ increases. Formally, we have

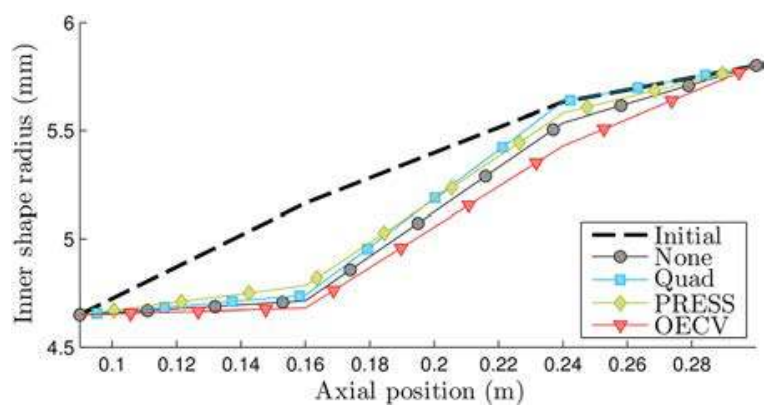

Fig. 7 Representation of the leadpipe inner radius along the instrument axis; the black dotted line to the initial geometry (measured on the Yamaha trumpet); each other line corresponds to the best design found by one of the four methods over 20 runs

$$
\hat{J}(\mathbf{x})=\frac{\sum_{j=1}^{p} w_{j}(\mathbf{x}) J\left(\mathbf{x}_{j}\right)}{\sum_{j=1}^{p} w_{j}(\mathbf{x})}, \text { where } w_{j}(\mathbf{x})=\phi\left(\left\|\mathbf{x}-\mathbf{x}_{j}\right\|_{2}\right)
$$

As for some of the RBF models, $\phi$ is a Gaussian kernel $\phi(d)=\exp \left(-\lambda^{2} d^{2}\right)$, where $\lambda$ is a shape parameter that controls the amount of smoothing in the model.

4.2.4 Selection of the Best Surrogate Model. Two error metrics are considered in this work to select the best surrogate model. The first metric is the leave-one-out cross-validation root mean square error (also named PRESS [31-33])

$$
E_{\mathrm{PRESS}}=\sqrt{\frac{1}{p} \sum_{i=1}^{p}\left(J\left(\mathbf{x}_{i}\right)-\hat{J}^{(-i)}\left(\mathbf{x}_{i}\right)\right)^{2}}
$$

where $\hat{J}^{(-i)}$ is the model built by leaving out the observation $\left[\mathbf{x}_{i}, J\left(\mathbf{x}_{i}\right)\right]$. This error metric allows to quantify not only the error of the model on the training point but also its predictive error. The second error metric, proposed by Ref. [25], is the leave-one-out cross-validation order error (OECV), which is based on the assumption that the error between $y$ and $\hat{J}$ is less important than the ability of the model to correctly order two candidates. This metric is defined as

$$
E_{\mathrm{OECV}}=\frac{1}{p^{2}} \sum_{i, j}\left(J\left(\mathbf{x}_{i}\right) \leq J\left(\mathbf{x}_{j}\right) \text { xor } \hat{J}^{(-i)}\left(\mathbf{x}_{i}\right) \leq \hat{J}^{(-j)}\left(\mathbf{x}_{j}\right)\right)
$$

where xor is the logical "exclusive or" operator. In other words, the error will be high if there are many couples $\left(\mathbf{x}_{i}, \mathbf{x}_{j}\right)$ for which the sign of $J\left(\mathbf{x}_{i}\right)-J\left(\mathbf{x}_{j}\right)$ is different from that of $\hat{J}^{(-i)}\left(\mathbf{x}_{i}\right)$ $-\hat{J}^{(-j)}\left(\mathbf{x}_{j}\right)$. The value of $E_{\text {OECV }}$ is bounded by $[0,1]$. We observe that if $E_{\mathrm{OECV}}>1 / 2$, then $\hat{J}$ is less accurate than its opposite function.

\section{Examples}

Two design problems are considered: one with two design optimization variables (2D) and one with five (5D). For the 2D problem, an exhaustive computation of the objective function on a fine discretization of the design space is tractable. It is therefore possible to assess the quality of the optimal solution with respect to the global optimum obtained using the exhaustive enumeration. The $5 \mathrm{D}$ problem corresponds to a more realistic design problem. For both problems, the initial guess $\mathbf{x}_{\text {init }}$ corresponds to the geometry of the Yamaha 6335 trumpet (measured using balls and calipers). The optimal bores are compared to this geometry to assess the quality of the results. However, the exact bore geometry of the Yamaha trumpet is not available due to proprietary issues. The comparison is thus made relative to an approximate measurement of this trumpet. Each problem is solved with a budget of 100 objective function evaluations, using the "true" physics-based sound simulation model, to investigate the performance of the optimization methodology at reasonable computational cost (approximately 5 CPU hours per run on a $3.4 \mathrm{GHz}$ Intel Core i72600 with $16 \mathrm{~GB}$ of RAM). Moreover, to allow for a reliable quantification of the efficiency of the optimization methods, each problem is solved 20 times with each of the four solvers.

5.1 Design Optimization Problem With Two Variables. The design variables concern two diameters of the leadpipe of a $\mathrm{Bb}$ trumpet, an important part of the bore that connects the mouthpiece to the tuning slide (see Fig. 2). This part, roughly conical, has a significant influence on the intonation and timbre of the instrument [10]. The impact of the two design variables on the geometry of the bore is illustrated in Fig. 7. The first adjustable 


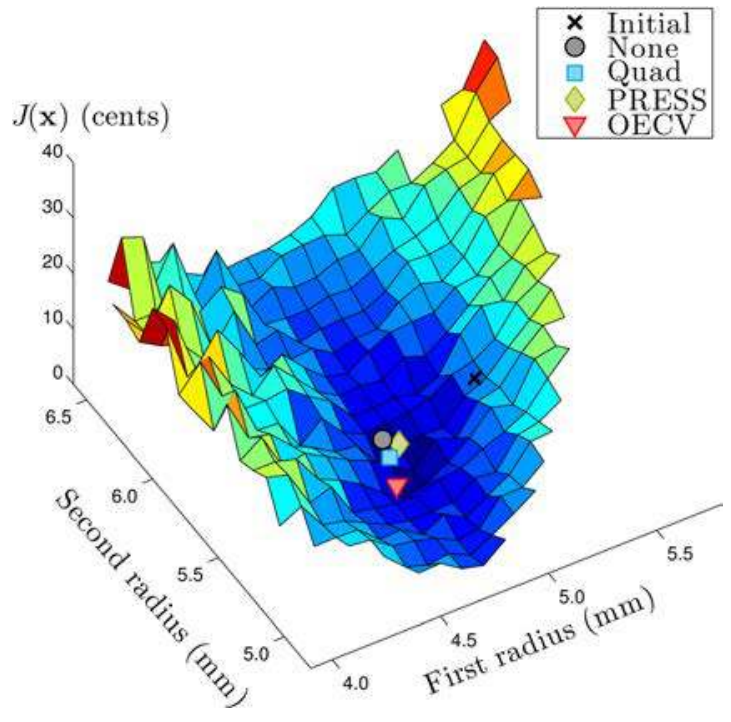

Fig. 8 Exhaustive computation of the objective function for the two-dimensional design example; the black cross denotes the initial geometry; the other dots denote the best solutions of the four employed strategies

radius (axial position of $0.16 \mathrm{~m}$ ) spans from $4 \mathrm{~mm}$ to $5.8 \mathrm{~mm}$ while the second (axial position of $0.24 \mathrm{~m}$ ) spans from $4.8 \mathrm{~mm}$ to $6.6 \mathrm{~mm}$. The rest of the trumpet corresponds to measurements made on a Yamaha 6335 trumpet with a Yamaha 15C4 mouthpiece (see Fig. 3 for the complete bore profile).

A single evaluation of the objective function $J(\mathbf{x})$ over the entire discretized design space (with a granularity of $0.01 \mathrm{~mm}$ ) is plotted in Fig. 8. The objective function has a global optimum of 3 cents at $\mathbf{x}=\left[\begin{array}{ll}4.8 & 5.5\end{array}\right]$ (in $\mathrm{mm}$ ). The maximum of $J(\mathbf{x})$ in the design space is 27 cents. The leadpipe geometry corresponding to the initial point (Yamaha trumpet) is represented by a black dotted line in Fig. 7. Its position on the objective function surface is denoted by the black cross on Fig. 8 .

Four optimization strategies are tested. The ones nicknamed "None" and "Quad" use the official NOMAD release 3.7.2 where the search step is omitted or where it employs local quadratic surrogate models, respectively. The strategies based on the use of the ensemble of surrogates tested with the two error metrics are named after the error metric, i.e., PRESS and OECV.

The history plot of the objective function for each optimization strategy is shown in Fig. 9.

On average, the best objective is obtained using OECV: detailed intonation improvements obtained with this strategy are shown in Fig. 10. The optimal objective function value for the best run of OECV is 0.9 cents at $\mathbf{x}=\left[\begin{array}{ll}4.68 & 5.43\end{array}\right]$ (in $\mathrm{mm}$ ). The

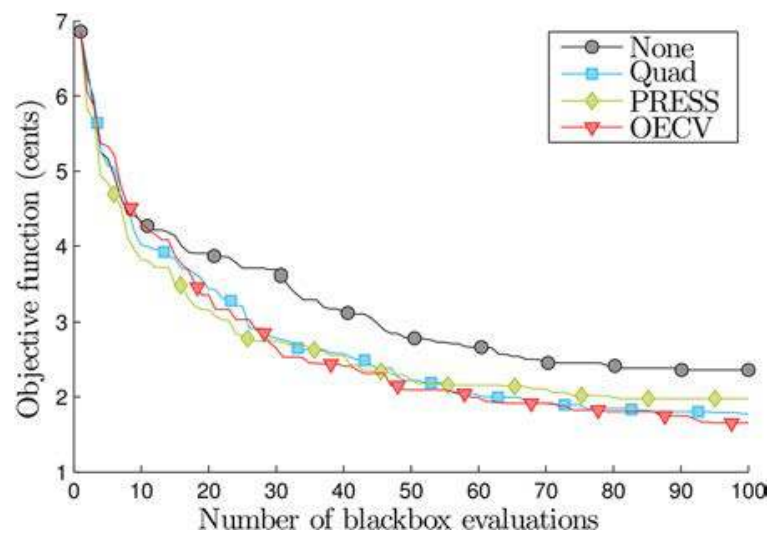

Fig. 9 Evolution of the objective in the 2D problem

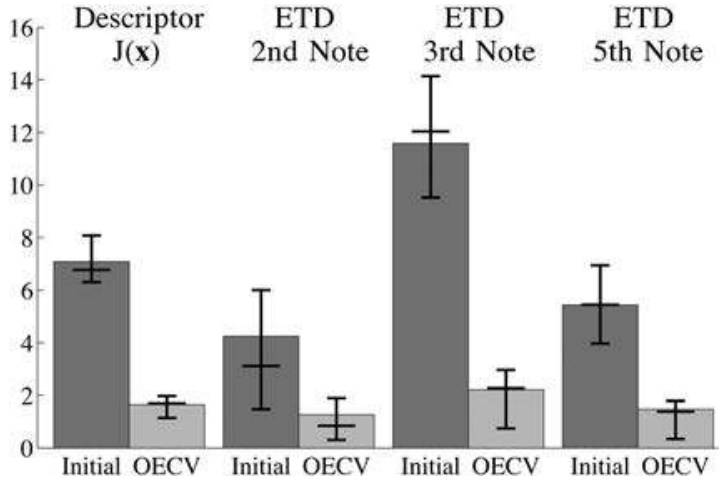

Fig. 10 Details of the intonation improvements obtained using the OECV method for the 2D case: the dark gray columns correspond to the initial geometry while the light gray columns correspond to the optimum; the two left columns are the objective function mean value while the six other columns represent the detailed mean absolute value of the ETDs; the black bars on each column correspond from bottom to top to the first quartile the median and the third quartile of distributions of the 20 runs

average intonation improvement is 5.4 cents, which is above the just noticeable difference (JND) in pitch ( 5 cents).

Note that the deviation of the fourth note is zero because this note is chosen as the tuning reference.

5.2 Design Optimization Problem With Five Variables. The five design variables concern the leadpipe and the mouthpiece. On the leadpipe, five parts of equal length $(l=44 \mathrm{~mm})$ are considered. The design variables are the inner radii of the leadpipe at the connection between two parts (four variables out of six control points because the initial and last control points are fixed to 4.8 and $5.6 \mathrm{~mm}$, respectively). These four inner radii values span from 3.8 to $6.6 \mathrm{~mm}$. The last variable corresponds to the depth of the mouthpiece (length of the cylinder before the cup of the mouthpiece) which spans from 0 to $6 \mathrm{~mm}$. The rest of the trumpet is as in the $2 \mathrm{D}$ example.

The performance of the four strategies is presented in Fig. 11 as for the $2 \mathrm{D}$ problem. On average, the OECV strategy yields the best design. The average optimal objective value is 1.5 cents, improving the overall intonation by 5.8 cents. The detailed intonation improvements obtained with this method are shown in Fig. 12 , and the final best optimum of the OECV strategy is $\mathbf{x}=[2.3$ 6.484 .135 .416 .42 ] (in $\mathrm{mm}$ ).

When the PRESS metric is used, the selected model error varies between 0.23 and 7.9, with an average of 3.1. For the OECV metric, the selected model error varies between 0 and 0.41 , with an average of 0.27 . For the OECV and PRESS strategies, we define the "model selection map" $S_{i, k}$ as the number of times that the

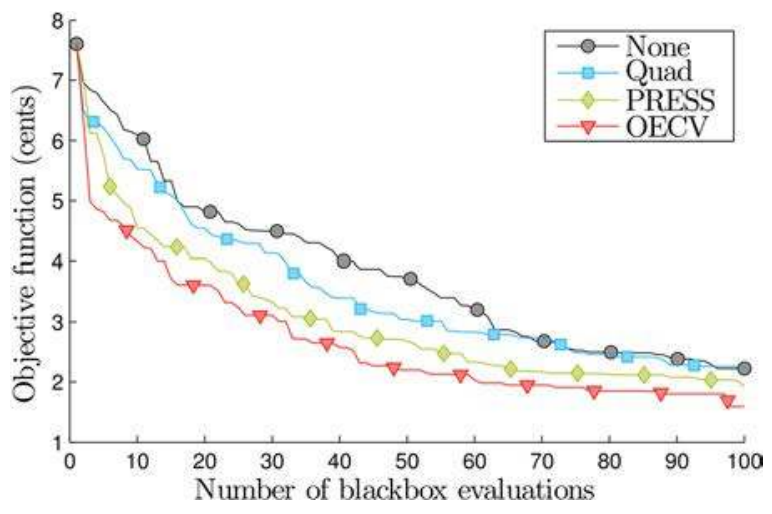

Fig. 11 Evolution of the objective in the 5D problem 
model $k$ (among the 17 models listed in Table 2) was selected at blackbox evaluation $i$ over the 20 runs. Figure 13 depicts the selection maps for these two strategies.

5.3 Discussion. The results are very promising and show significant improvements in the intonation of the instrument in both cases. The solution of the 2D problem improves the objective by up to $75 \%$ (average value for the OECV solver). For the 5D problem, the objective is improved by up to $79 \%$ (average value for the OECV solver). The optimal designs of the 2D problem are close to the global minimizer as can be seen in Fig. 8. This demonstrates that the algorithm formulations converge toward the minimum (recall that the computation budget was limited to 100 "true" model function evaluations). The ensemble surrogate strategy with OECV is the most successful in both cases though this advantage is very mild for the $2 \mathrm{D}$ problem. From an acoustic point of view, it is worth noting that both for the $2 \mathrm{D}$ and for the $5 \mathrm{D}$ problem, the optimum improves the intonation of every note. The optimal designs for the 2D problem are plausible. For the 5D problem, the optimum (best design of the OECV method over 20 runs) is counterintuitive since the leadpipe does not have a positive slope along the whole trumpet axis (the diameter at the first control point, $6.48 \mathrm{~mm}$, is larger than at the previous and next control point, respectively, $4.8 \mathrm{~mm}$ and $4.13 \mathrm{~mm}$ ). This kind of form for a leadpipe is not common among trumpets because they are very difficult to manufacture. We see thus that the optimization algorithm was able to explore the design space in order to find exotic designs, which significantly improve instrument intonation. Finally, the three additional degrees-of-freedom considered in the 5D problem improve the capability of the algorithm to find a slightly better optimum.

From an optimization point of view, it is interesting to observe that the PRESS and OECV metrics lead to different models being selected. The use of the PRESS metric strongly favors the PRS models of degree 1 and 2 with regularization and the models KS 1 and KS 3. The OECV metrics favor a more diverse model selection, i.e., the use of Kernel smoothing with a high shape parameter (KS 10), and the use of RBF models, which are nearly ignored when the PRESS metric is used. It is also interesting to see that, for both metrics, the shape parameter of the selected Kernel smoothing models tends to increase, which implies the ability to model more local variations as the number of data points increases. The fact that OECV metric leads to a more diverse model selection and to more efficient optimization makes it an interesting option for surrogate-assisted optimization.

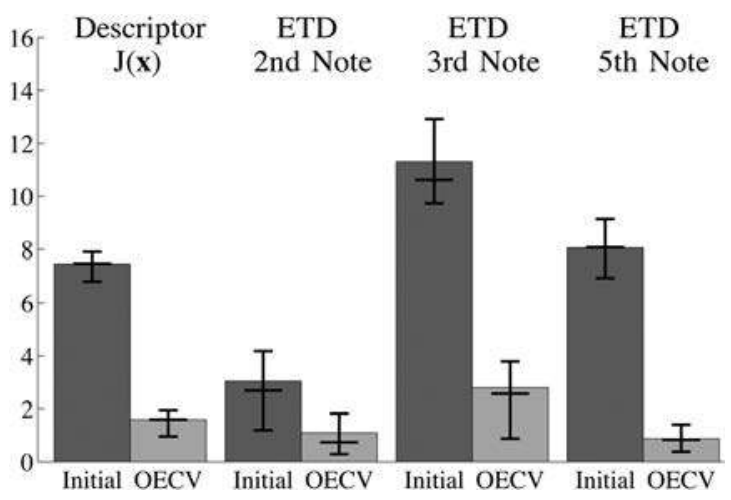

Fig. 12 Details of the intonation improvements obtained using the OECV method for the 5D case: the dark gray columns correspond to the initial geometry while the light gray columns correspond to the optimum; the two left columns are the objective function mean value while the six other columns represent the detailed mean absolute value of the ETDs; the black bars on each column correspond from bottom to top to the first quartile the median and the third quartile of distributions of the 20 runs

\section{Conclusions}

This work proposes a new paradigm for design optimization of brass instruments. The originality of the approach lies in the fact that the objective function is not limited to a characterization of the instrument alone, but is based on the complex interaction between the instrument and the musician. The main challenge consists in the integration of physics-based simulations in an iterative optimization loop, which requires that simulations converge toward auto-oscillations for every considered point of the design space. This property is ensured by means of a preprocessing step of the virtual embouchures of the musician and a stochastic approach that assesses the objective function using a Monte Carlo method. The sound simulations are conducted using a physicsbased model that relies on the harmonic balance technique. This allows to define the objective function by computing the global

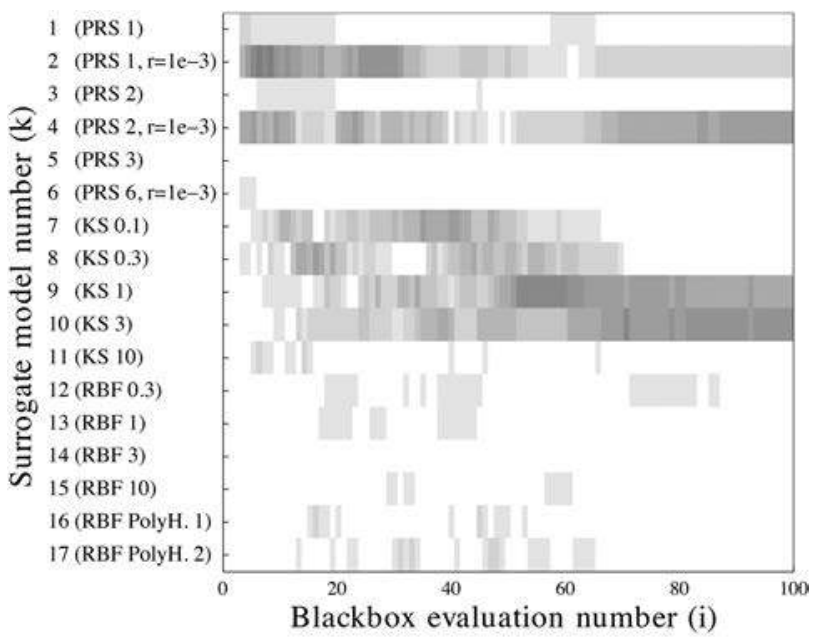

(a)

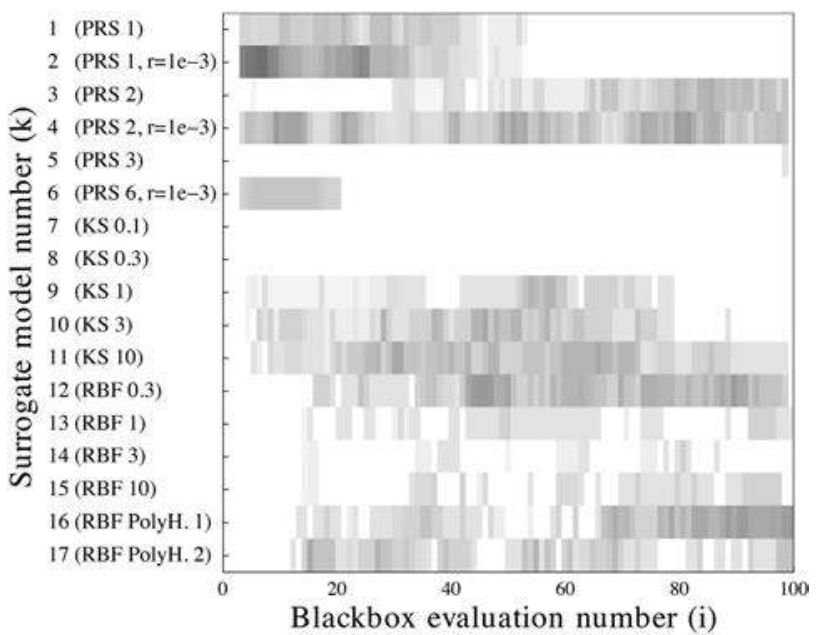

(b)

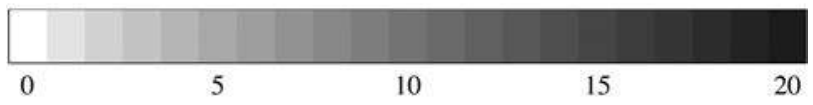

(c)

Fig. 13 Model selection map $S_{i, k}$ for PRESS and OECV strategies on the 5D problem; the upper (respectively, lower) map indicates which models were selected with the PRESS (respectively, OECV) error metric; the gray intensity indicates how often the model $\mathbf{k}$ was selected during blackbox evaluation $i$ over the 20 runs; darker gray indicates a model selected more frequently (a) selection with PRESS metric, $(b)$ selection with OECV metric, and (c) legend: never selected always selected 
instrument's intonation for a set of different virtual embouchure. The MADS optimization algorithm is used, and different formulations of the surrogate-based search step have been tested to improve its efficiency. The results are encouraging both from an acoustics and from an algorithmic point of view. The intonation improvements are significant, and the obtained optimal designs are rational.

This work can be extended in many directions. Regarding acoustics, more design variables can be considered, in particular with respect to the mouthpiece. One could modify its thinnest radius and the length proportion between the hemisphere and the divergent cone. Another direction considers the objective function. Since a sound in a permanent regime provides us with the amplitudes of the harmonics, objective functions based on the instrument timbre can be formulated. The influence of some embouchure parameters on the optimal solutions can also be considered, for example the intonation variations under a modification of the embouchure's dynamics. As several sound quality descriptors are available, a bi-objective optimization or an optimization of one descriptor subject to constraints based on some other descriptors would be a natural consideration. In particular, it would be interesting to investigate a timbre descriptor under an intonation constraint, because trumpeters may be able to accept a slightly out-of-tune trumpet if its "global" sound is outstanding. Concerning the optimization process, it may be interesting to use the two metrics "PRESS" and "OECV" for aggregating different models instead of selecting only one. Moreover, if constrained problems are considered, an ensemble of models may be very effective for selecting different surrogates for different functions (objective and constraints).

\section{Acknowledgment}

The authors are grateful to the NOMAD team (Professor Charles Audet, Professor Sébastien Le Digabel and Dr. Christophe Tribes), Professor Said Moussaoui for his help with the optimization problem formulation, and Professor Jean-Pierre Dalmont for his precious help with the input impedance models. B. Talgorn and M. Kokkolaras are grateful for the partial support of FRQNT Grant No. 2015-PR-182098; this support does not constitute an endorsement of the opinions expressed in this paper.

\section{References}

[1] Fritz, C., and Dubois, D., 2015, "Perceptual Evaluation of Musical Instruments: State of the Art and Methodology," Acta Acust. Acust., 101(2), pp. 369-381.

[2] Campbell, M., 2004, "Brass Instruments as We Know Them Today," Acta Acust. Acust., 90(4), pp. 600-610.

[3] Causse, R., Kergomard, J., and Lurton, X., 1984, "Input Impedance of Brass Musical Instruments: Comparisons Between Experiment and Numerical Models," J. Acoust. Soc. Am., 75(1), pp. 241-254.

[4] Benade, A. H., 1966, "Relation of Air-Column Resonances to Sound Spectra Produced by Wind Instruments," J. Acoust. Soc. Am., 40(1), pp. 247-249.

[5] Kausel, W., 2001, "Optimization of Brasswind Instruments and its Application in Bore Reconstruction," J. New Music Res., 30(1), pp. 69-82.

[6] Braden, A. C. P., Newton, M. J., and Campbell, D. M., 2009, "Trombone Bore Optimization Based on Input Impedance Targets,” J. Acoust. Soc. Am., 125(4), pp. 2404-2412.

[7] Noreland, D., 2013, "A Gradient Based Optimisation Algorithm for the Design of Brass-Wind Instruments," Ph.D. thesis, Uppsala University, Uppsala, Sweden.
[8] Poirson, E., Petiot, J. F., and Gilbert, J., 2007, "Integration of User Perceptions in the Design Process: Application to Musical Instrument Optimization," ASME J. Mech. Des., 129(12), pp. 1206-1214.

[9] Eveno, P., Petiot, J. F., Gilbert, J., Kieffer, B., and Causse, R., 2014, "The Relationship Between Bore Resonance Frequencies and Playing Frequencies in Trumpets," Acta Acust. Acust., 100(2), pp. 362-374.

[10] Petiot, J. F., and Gilbert, J., 2013, "Comparison of Trumpets' Sounds Played by a Musician or Simulated by Physical Modelling," Acta Acust. Acust., 99(4), pp. 629-641.

[11] Woodhouse, J., Manuel, E. K. Y., Smith, L. A., Wheble, A. J. C., and Fritz, C., 2012, "Perceptual Thresholds for Acoustical Guitar Models," Acta Acust. Acust., 98(3), pp. 475-486.

[12] Gilbert, J., Kergomard, J., and Ngoya, E., 1989, "Calculation of the SteadyState Oscillations of a Clarinet Using the Harmonic Balance Technique," J. Acoust. Soc. Am., 86(1), pp. 35-41.

[13] Dalmont, J. P., and Le Roux, J. C., 2008, "A New Impedance Sensor for Wind Instruments,” J. Acoust. Soc. Am., 125(5), p. 3014.

[14] Tournemenne, R., Petiot, J. F., and Gilbert, J., 2016, "The Capacity for Simulation by Physical Modeling to Elicit Perceptual Differences Between Trumpet Sounds," Acta Acust. Acust., 102(6), pp. 1072-1081.

[15] Cullen, J., Gilbert, J., and Campbell, M., 2000, "Brass Instruments: Linear Stability Analysis and Experiments With an Artificial Mouth," Acta Acust. Acust., 86(4), pp. 704-724.

[16] Fletcher, N. H., and Tarnopolsky, A., 1999, "Blowing Pressure, Power, and Spectrum in Trumpet Playing," J. Acoust. Soc. Am., 105(2), pp. 874-881.

[17] Audet, C., and Dennis, Jr., J. E., 2006, "Mesh Adaptive Direct Search Algorithms for Constrained Optimization," SIAM J. Optim., 17(1), pp. 188-217.

[18] Audet, C., Ianni, A., Le Digabel, S., and Tribes, C., 2014, "Reducing the Number of Function Evaluations in Mesh Adaptive Direct Search Algorithms," SIAM J. Optim., 24(2), pp. 621-642.

[19] Le Digabel, S., 2011, "Algorithm 909: NOMAD: Nonlinear Optimization With the MADS Algorithm," ACM Trans. Math. Software, 37(4), pp. 44:1-44:15.

[20] Booker, A. J., Dennis, Jr., J. E., Frank, P. D., Serafini, D. B., Torczon, V., and Trosset, M. W., 1999, "A Rigorous Framework for Optimization of Expensive Functions by Surrogates," Struct. Multidiscip. Optim., 17(1), pp. 1-13.

[21] Talgorn, B., Le Digabel, S., and Kokkolaras, M., 2015, "Statistical Surrogate Formulations for Simulation-Based Design Optimization," ASME J. Mech. Des., 137(2), p. 021405.

[22] Goel, T., Haftka, R. T., Shyy, W., and Queipo, N. V., 2007, "Ensemble of Surrogates," Struct. Multidiscip. Optim., 33(3), pp. 199-216.

[23] Viana, F. A. C., Haftka, R. T., Steffen, J. V., Butkewitsch, S., and Leal, M. F., 2008, "Ensemble of Surrogates: A Framework Based on Minimization of the Mean Integrated Square Error," 49th AIAA/ASME/ASCE/AHS/ASC Structures, Structural Dynamics, and Materials Apr. 7-10, Schaumburg, IL.

[24] Acar, E., and Rais-Rohani, M., 2008, "Ensemble of Metamodels With Optimized Weight Factors," Struct. Multidiscip. Optim., 37(3), pp. 279-294.

[25] Audet, C., Kokkolaras, M., Le Digabel, S., and Talgorn, B., 2016, "OrderBased Error for Managing Ensembles of Surrogates in Derivative-Free Optimization," Les Cahiers du GERAD, Technical Report No. G-2016-36.

[26] Müller, J., and Piché, R., 2011, "Mixture Surrogate Models Based on Dempster-Shafer Theory for Global Optimization Problems," J. Global Optim., 51(1), pp. 79-104.

[27] Queipo, N., Haftka, R., Shyy, W., Goel, T., Vaidyanathan, R., and Tucher, P., 2005, "Surrogate-Based Analysis and Optimization," Prog. Aerosp. Sci., 41(1), pp. 1-28.

[28] Hastie, T., Tibshirani, R., and Friedman, J., 2001, The Elements of Statistical Learning, (Springer Series in Statistics), Springer, Berlin.

[29] Buhmann, M., 2003, Radial Basis Functions: Theory and Implementations, (Cambridge Monographs on Applied and Computational Mathematics), Cambridge University Press, New York.

[30] Viana, F. A., Haftka, R. T., and Watson, L. T., 2013, "Efficient Global Optimization Algorithm Assisted by Multiple Surrogate Techniques," J. Global Optim., 56(2), pp. 669-689.

[31] Goel, T., and Stander, N., 2009, "Comparing Three Error Criteria for Selecting Radial Basis Function Network Topology," Comput. Methods Appl. Mech. Eng., 198(27-29), pp. 2137-2150.

[32] Varma, S., and Simon, R., 2006, "Bias in Error Estimation When Using CrossValidation for Model Selection," BMC Bioinf., 7(1), p. 91.

[33] Viana, F. A. C., Picheny, V., and Haftka, R. T., 2009, "Conservative Prediction Via Safety Margin: Design Through Cross-Validation and Benefits of Multiple Surrogates," ASME Paper No. DETC2009-87053. 